\title{
Fingerprint Recognition Using Principal Gabor Basis Function
}

\author{
Chih-Jen Lee, Sheng-De Wang, and Kuo-Ping Wu
}

\author{
Dept. of Electrical Engineering, National Taiwan University, Taipei 106, Taiwan, China \\ Email: cjlee,sdwang,wgb@hpc.ee.ntu.edu.tw
}

\begin{abstract}
Based on the Gabor transform, we propose a unified viewpoint for fingerprint image representation and recognition. In a local fingerprint image, we demonstrate the inter-ridge distances and ridge directions can be obtained by principal Gabor basis function $(G B F)$ directly. Besides, according to the locations of principal $G B F$ on the spatial-frequency plane, the similarities of two local ridge structures can be measured easily. As compared with the global Gabor-based approach, the proposed method not only saves memory space, but also reaches higher recognition rate.
\end{abstract}

\section{INTRODUCTION}

Based on uniqueness, durability, and convenience, fingerprints have been widely applied in several fields of personal identification such as access control, criminal investigation, and Internet authentication. Fingerprint patterns are full of ridges and valleys and these structures provide essential information for matching, recognition, and classification. Conventionally, most researchers use minutiae, a group of ridge endings and bifurcations, as the features of fingerprint patterns [1] Unfortunately, the minutia-based approach contains many processing steps and these steps are almost time consumption. In addition, this approach relies heavily on the quality of input images and the accuracy of every step. In fingerprint images, however, minutiae are not always clear even though the information of ridge directions and inter-ridge distances is preserved.

Gabor filters, simulated visual vertex cells, have the properties of spatial localization, orientation selectivity, and spatial-frequency selectivity [2]. Therefore, Gabor filters have been applied successfully and widely to many fields. There are mainly two categories. One is image reconstruction; images are represented by a complete or incomplete Gabor basis set. Its purpose is image compression [3]. Another is image analysis; only a few suitable and important Gabor filters are used to detect special features, such as texture classification [4], face recognition [5], handwritten character recognition [6], fingerprint classification [7], and fingerprint recognition [8][9]. In [7]-[9], Gabor-based features were proposed as the fingerprint features to avoid the disadvantages of the minutiae-based approach. Jain et al. used real Gabor filters as their features for fingerprint classification and recognition, but this approach must convolve each pixel by the real components of Gabor filters in an image to avoid the shift problem. This process wasted major time to the overall feature extraction. In [9], we demonstrated that only the magnitude Gabor features could be used as the features of fingerprint patterns when each block was sampled once. That is, the magnitude Gabor features can avoid the shift problem and reduce the computation time. To obtain a satisfactory recognition result, nonetheless, selecting a suitable bank of Gabor filters is very important to the Gaborbased approach. Unfortunately, this approach needs a lot of time to tune empirically the parameters of Gabor filters by global consideration.

In fact, fingerprint patterns contain various ridge directions and inter-ridge distances in each local region. That is, each local fingerprint has its particular ridge direction and spatial-frequency. In order to capture these intrinsic characteristics of ridge structures, we propose a local Gabor-based approach to determine the suitable Gabor filters by using only local information. In addition, we transform each local fingerprint image into Gabor representation, a combination of the complete Gabor basis functions (GBFs), to find out the most suitable Gabor filter. For further feature reduction, the selected Gabor filter is mapped to an index of the complete GBFs. At last, we also compare GBFs in the spatial-frequency domain to illustrate the similarities of ridge structures and analyze the feasibility of the proposed method to test a small-scale access control system.

\section{FINGERPRINT IMAGES AND GABOR BASIS FUNCTIONS (GBFS)}

\subsection{Power spectra of fingerprint images}

At first, we observe the intrinsic characteristics of fingerprint patterns in the spatial and spatialfrequency domains. In a local fingerprint image, the ridge directions and inter-ridge distances are similar. Fig. 1(a) and Fig. 2(a) show some regions of fingerprint images. To analyze the phenomenon of fingerprint images in the spatial-frequency domain, we transfer these images by Fourier transform and observe their power spectra. Before doing Fourier transform, we subtract the DC value of the region from every pixel to focus on the power spectra of the $\mathrm{AC}$ components. In Fig. 1(a), from left to right, the ridge directions are $0^{\circ}, 45^{\circ}, 90^{\circ}$, and $135^{\circ}$, respectively. The corresponding $2-\mathrm{D}$ power spectra, 
shown in Fig. 1(b), have two high peaks and these peaks are symmetric to the origin. In addition, different orientations have different locations of the twin peaks relative to the origin on the spatialfrequency plane. Fig. 2(a) shows some different interridge distances with the same orientation. The corresponding power spectra are shown in Fig. 2(b). From Fig. 2(b), the power spectra also have twin peaks and the angles from the centers of these peaks relative to the origin are similar on the spatialfrequency plane. On the contrary, the distances from the centers of these peaks to the origin are decreasing when the inter-ridge distances are increasing. According to the characteristics of the angle and the distance from the centers of the peaks to the origin on the spatial-frequency plane, the ridge direction and the inter-ridge distance of the spatial domain can be estimated easily. That is, the intrinsic characteristics of fingerprint images are easily captured in the spatial-frequency domain rather than the spatial domain.

\subsection{Gabor expansion, Gabor responses, and principal GBF}

The 2-D complete set of GBFs can be expressed as:

$$
\begin{aligned}
G_{p q r s}(x, y)= & \exp \left\{-\left[(x-p)^{2}+(y-q)^{2}\right] / \sigma^{2}\right\} \\
& \exp \left[2 \pi j(x r+y s) / N_{f}\right]
\end{aligned}
$$

where $j=\sqrt{-1}, p, q=0,1, \cdots, N_{s}-1$, and

$r, s=-N_{f} / 2+1,-N_{f} / 2+2, \cdots,-1,0,1, \cdots, N_{f} / 2-1, N_{f} / 2$. In the spatial domain, $N_{s}$ is the number of spatial samples, $(p, q)$ is the spatial window center, and $\sigma$ decides the extent of spatial windows. In the spatialfrequency domain, $N_{f}$ is the number of spatialfrequency samples and $(r, s)$ is the location of the frequency center. The angle $\theta$ and radial frequency $f$ of GBF are determined by $\tan ^{-1}(s / r)$ and $\sqrt{\left(s / N_{f}\right)^{2}+\left(r / N_{f}\right)^{2}}$, respectively. Their relationship on the spatial-frequency plane is demonstrated in Fig. 3. In Eq. 1, we set the number of spatial samples, the number of spatial-frequency samples, and the extents of spatial windows along $x$ and $y$ axes as the same values for simplification. In the spatial domain, the real components of these basis functions for $N_{s}=N_{f}=$ 16 are shown in Fig. 4. From Fig. 4, the orientationselective properties of the GBFs are obvious.

In the spatial-frequency domain, each real GBF (except $r=s=0$ ) has twin Gaussian peaks. The envelope of Gaussian function, which is proportional to the reciprocal of $\sigma$, determines the channel bandwidths. Various orientations are shown in Fig. 5 (corresponding to $0^{\circ}, 45^{\circ}, 90^{\circ}$, and $135^{\circ}$ ) and the radial frequencies, the distance from center of the Gaussian function to the origin, are increasing from left to right.
Although we only demonstrate some directions in Fig 5 , in fact, each GBF has twin Gaussian peaks at its frequency center $(r, s)$. The complete GBFs spread $N_{f}$ $\times N_{f}$ Gaussian peaks on the spatial-frequency plane. They only respond to the image with the same orientation and radial frequency as narrowband filters.

A raw image $I(x, y)$ can be expressed as a combination of the complete 2-D GBFs. The Gabor expansion equation is

$I(x, y)=\sum_{p} \sum_{q} \sum_{r} \sum_{s} c_{p q r s} G_{p q r s}(x, y)$

where $c_{p q r s}$ is the Gabor coefficients indicating their importance for image reconstruction. Unfortunately, the computational complexity of Gabor coefficients comes from the non-orthogonality of the GBFs. The magnitudes of Gabor coefficients for various ridge directions and inter-ridge distances are shown in Fig. 1(c) and 2(c). For visualization, the highest response of the corresponding GBF is mapped to the highest gray value.

The Gabor response $g$ of each GBF corresponding $(r, s)$ is defined as follows:

$g(r, s)=\left|\sum_{p}^{N_{s}} \sum_{q}^{N_{s}} I(x, y) G_{p q r s}(x, y)\right|$

where $I$ is a $N_{s} \times N_{s}$ input image. Fig. 1(d) and 2(d) show the corresponding Gabor responses from Fig. 1(a) and 2(a) after removing the DC value of every pixel in the region. From Fig. 1(d) and Fig. 2(d), the Gabor responses also have twin peaks, and the corresponding locations are similar to their Fourier power spectra and Gabor coefficients. This means that the two corresponding GBFs (in fact, they are the same) have the highest responses to the local fingerprint image. Moreover, the orientation and spatial-frequency of the corresponding GBF can represent mainly the local region because the image energy concentrates at its frequency. In other words, a local fingerprint image can easily be captured the main characteristics by using only one GBF. We name it as the principal GBF of the local region.

\section{FINGERPRINT RECOGNITION}

\subsection{Feature vectors}

Because the principal GBF can exactly capture the orientation and spatial-frequency of a local ridge structures, the input dimension is reduced from pixels to only one GBF. A GBF, determined by $(r, s)$, can also be reduced to an index of the complete GBFs. If a local region has $16 * 16$ pixels, for example, then there are 256 GBFs and the index of the principal GBF needs only one byte. That is, the input feature vectors are reduced by a factor of 256 .

If two fingerprint patterns are collected from the same fingerprint, the ridge directions and inter-ridge 
distances of the corresponding local regions in fingerprint images are very similar. In other words, the locations of the corresponding principal GBFs are quite near on the spatial-frequency plane, even though there is a little rotation. That is, we can differentiate their similarities from the distance of the corresponding principal GBFs on the spatialfrequency plane. Moreover, the $(r, s)$ of the principal GBF just reflects its location on the spatial-frequency plane. Although the principal GBF is stored as an index value, it is very easily converted to $(r, s)$ by division. Therefore, we adopt a set of $(r, s)$ values from every local fingerprint images as the input feature vectors for recognition.

\subsection{The proposed approach}

The whole procedure, including only three processes, is shown in Fig. 6. Comparing two fingerprint patterns must be based on the same reference point. We use the core point, the topmost point on the innermost upward recurving ridge in fingerprint, as the reference point. To avoid the error caused by the core point detection algorithm, we point out the core points of all fingerprint patterns manually. Based on the core point, we crop the fingerprint image into $96 \times 112$ pixels and divide the cropped image into a set of $16 \times 16$ non-overlapping regions. According to the maximum Gabor responses to find the principal GBFs of all regions, $6 \times 7(r, s)$ values are obtained for each fingerprint image. In the recognition stage, we select single nearest neighbor $(\mathrm{NN})$ classifier to avoid gaining benefits from the classifier. This is because we aim at analyzing the influence for the new features of fingerprints in recognition.

\section{EXPERIMENTAL RESULTS}

We collect fingerprint patterns with various ink pressures from 16 persons. Each person pressed his right thumb finger 12 times on a piece of paper. Therefore, our fingerprint database contains 192 inked fingerprint patterns scanned at $200 \mathrm{dpi}$. Although the fingerprint databases of NIST and FBI are sampled at $500 \mathrm{dpi}$, the fingerprint images can be recognized at $200 \mathrm{dpi}$ by human eyes. Recognizing low quality images is efficient and practicable for a small-scale fingerprint recognition system.

In single $\mathrm{NN}$ experiments, $k$ patterns per individual are selected as the training database ( $16 k$ patterns) and the rest $16 \times(12-k)$ patterns as the test database. In the global approach, we use four directions and set the radial frequencies as the reciprocal of the average inter-ridge distance to extract the Gabor responses for all regions. Except directions and radial frequencies, the other parameters are set the same with the proposed approach. The results of recognition rates with no rejection option are shown in Table 1 . From Table 1, we find that the recognition rate of the proposed method with only two patterns per individual is higher than $95 \%$ and all situations are superior to the global approach.

Besides the recognition rates are improved, the storage for the features of fingerprint patterns is also reduced. In the global approach, a bank of Gabor responses, which are floating point values, is stored for a local region. Nonetheless, only an unsigned integer index value is stored for the principal GBF in the local approach.

\section{CONCLUSIONS}

We develop a local Gabor-based approach to extract the intrinsic characteristics of a local fingerprint image for recognition. The proposed method illustrates that the principal GBF can capture the main characteristics of ridge orientation and ridge frequency when the raw image is transformed into the Gabor space. Therefore, the feature dimension of a local region can be reduced to an index value. On the spatial-frequency plane, according to the locations of the principal GBFs, we can measure the similarities of two local ridge structures easily. Moreover, the recognition rates are also better than the global approach. In conclusion, the proposed approach is efficient and feasible.

\section{REFERENCES}

[1] A.K. Jain, L. Hong, and R. Bolle, "On-line fingerprint verification," IEEE Trans. Pattern Analysis Machine Intelligent, vol. 19, no. 4, pp. 302-314, 1997.

[2] J.G. Daugman, "Uncertainty relation for resolution in space, spatial frequency, and orientation optimized by two-dimensional visual cortical filters," J. Opt. Soc. Amer. A, vol. 2, no. 7, pp. 1160-1169, 1985.

[3] P. Lau and N.P. Papanikolopoulos, "Adaptive Gabor image transformation, application in scientific visualization," Proceedings of International Conference on Image Processing, vol. 3, pp. 504-507, 1995.

[4] T.P. Weldon, W.E. Higgins, and D.F. Dunn, "Efficient Gabor filter design for texture segmentation," Pattern Recognition, vol. 29, no. 12, pp. 2005-2015, 1996.

[5] L.Wiskott, J.M. Fellous, N. Krüger, and C. von der Malsburg, "Face recognition by elastic brunch graph matching," IEEE Transactions on Pattern Analysis and Machine Learning, vol. 19, No. 7, pp. 775-779, 1997.

[6] Y. Hamamoto, S. Uchimura, M. Watanabe, T. Yasuda, Y. Mitani, and S. Tomita, "A Gabor filter-based method for recognizing handwritten numerals," Pattern Recognition, vol. 31, no. 4, pp. 395-400, 1998.

[7] A.K. Jain, S. Prabhakar, and L. Hong, "A multichannel approach to fingerprint classification," IEEE Transactions on Pattern 
Analysis Machine Intelligent, vol. 21, no. 4, pp. 348-359, 1999.

[8] A.K. Jain, S. Prabhakar, L. Hong, and S. Pankanti, "Filterbank-based fingerprint matching," IEEE Transactions on Image Processing, vol. 9, no. 5, pp. 846-859, 2000.

[9] C.J. Lee and S.D. Wang, "Fingerprint feature extraction using Gabor filters," Electronics Letters, vol. 35, no. 4, pp. 288-290, 1999.

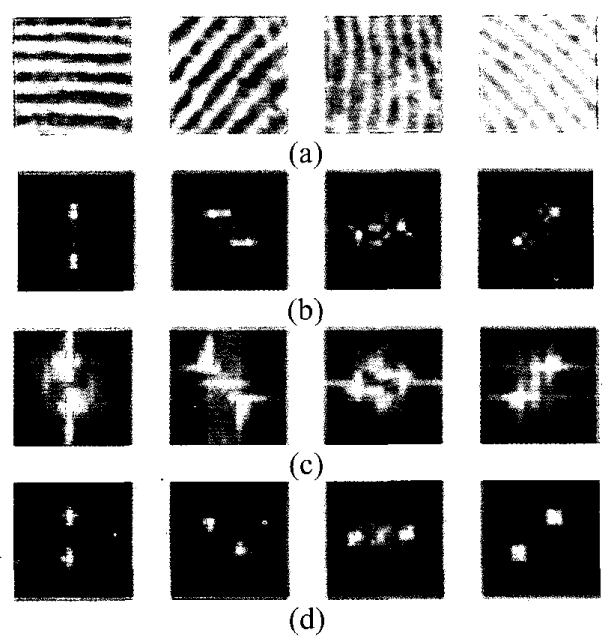

Fig. 1 (a) Original images and corresponding (b) power spectra (c) coefficients and (d) responses of GBFs for various ridge directions.
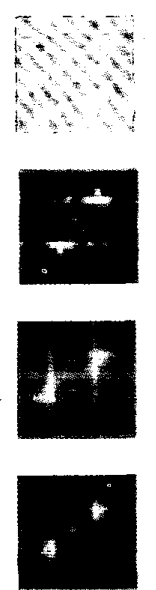
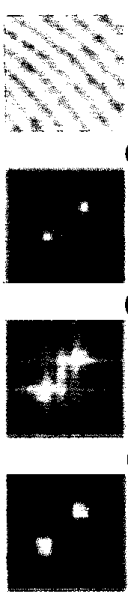

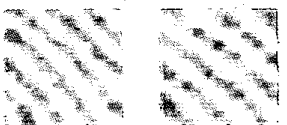

(a)

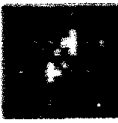

(b)

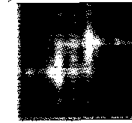

(c)

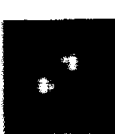

(d)
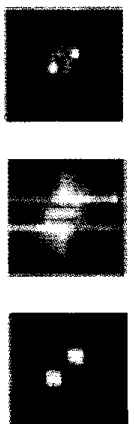

Fig. 2 (a) Original images and corresponding (b) power spectra (c) coefficients and (d) responses of GBFs for various ridge frequencies.

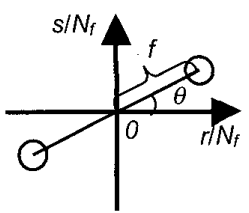

Fig. 3 Parameters of GBF on the spatial-frequency plane.

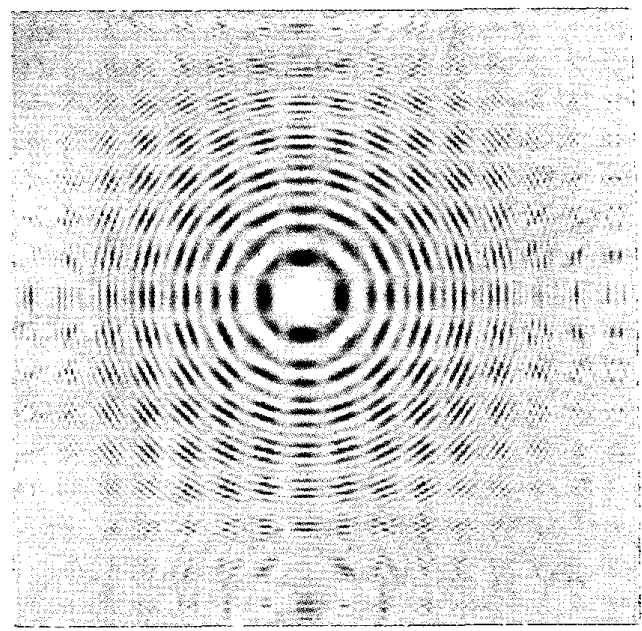

Fig. 4 Real components of complete Gabor basis functions.

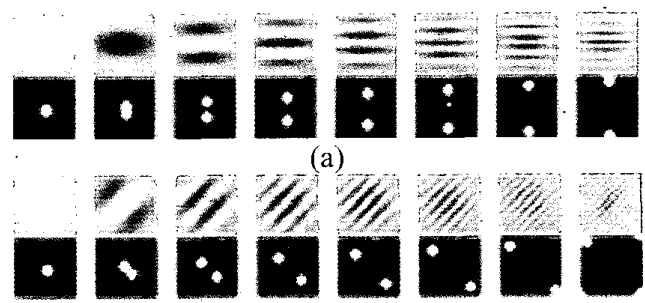

(b)

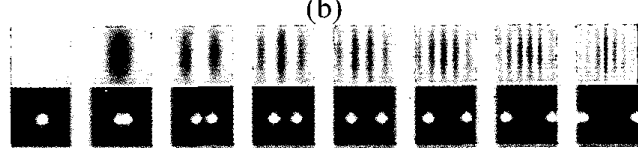

(c)

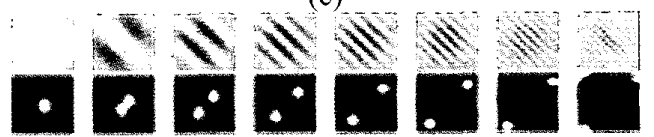

(d)

Fig. 5 Power spectra of Gabor filters corresponding to (a) $0^{\circ}$, (b) $45^{\circ}$, (c) $90^{\circ}$, and (d) $135^{\circ}$ (the radial frequencies increase from left to right).

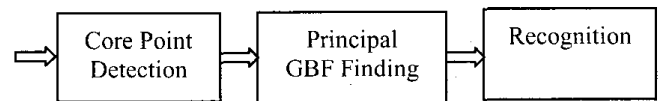

Fig. 6 Block diagram of the proposed method.

\begin{tabular}{|c|c|c|c|}
\hline$k$ patterns/person & 1 & 2 & 3 \\
\hline Local & 90.9 & 95.6 & 97.9 \\
\hline Global & 88.6 & 93.8 & 96.5 \\
\hline
\end{tabular}

Table 1 Results of recognition rate (\%). 